\title{
Climate and Volume and Blood Pressure Trends in Taiwanese Hemodialysis Patients
}

\author{
Hugh Feidhlim Woods ${ }^{c}$ Chen Ting Cheng ${ }^{a}$ Po Sung Lim ${ }^{b}$

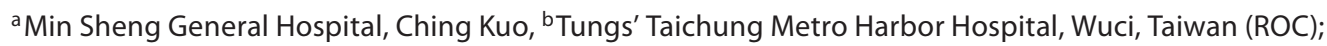 \\ 'Fresenius Medical Care Asia Pacific, Hong Kong, SAR (China)
}

We read with interest the recent paper by Hwang et al. [1] from Taiwan describing changes in fluid gain related to seasonal climate change in their HD patients. We have also noted seasonal changes in volume and blood pressure status in the patients treated at our two hospitals, one (Min Shen) situated in the North of Taiwan and the other (Tung Hospital) situated in central Taiwan.

During 2007, 496 patients received maintenance HD in the five dialysis clinics associated with our hospitals. As part of the continuous audit of dialysis clinics af- filiated with Fresenius Medical Care (Nephrocare, Taiwan), we submit to a central database the data related to index dialysis treatments on a monthly basis. For the 496 patients, we had complete data for 4,330 treatments (2,254 from Min Shen and 2,076 from Tung). The characteristics of the patients and selected data averages and dispersions are shown in table 1.

Like Hwang, we categorized our climate into the hot season, the cold season and months with intermediate climate. Using the weight loss from ultrafiltration as a surrogate for interdialytic weight gain and expressing this as a fraction of postdialysis weight, we confirm the seasonal patterns described by Hwang as shown in figure 1. Fractional weight loss at $4.5 \%$ during the cold season was significantly higher than the $4.3 \%$ recorded during the hot season (ANOVA: $\mathrm{p}=0.004$ ) and neither was significantly different to fractional weight loss during the intermediate months.

We also identified higher mean predialysis systolic and mean arterial pressures and a greater average drop in postdialysis systolic BP as shown in figure 2. As with Hwang's patients this seasonal effect was
Table 1. Characteristics of treated patients

\begin{tabular}{lrrr}
\hline & \multicolumn{3}{c}{ Selected characteristics of patients } \\
\cline { 2 - 4 } & minimum & maximum & mean $\pm \mathrm{SD}$ \\
\hline Age at test time, years & 18.83 & 93.28 & $61.48 \pm 12.67$ \\
Pre-weight, kg & 34.70 & 107.63 & $61.04 \pm 11.79$ \\
Post-weight, kg & 33.10 & 103.00 & $58.48 \pm 11.37$ \\
Weight loss, kg & 0.00 & 5.90 & $2.56 \pm 1.07$ \\
UF fraction weight & 0.00 & 0.16 & $0.04 \pm 0.02$ \\
Sodium, mEq/l & 120.00 & 154.00 & $137.61 \pm 3.48$ \\
Albumin, g/l & 21.00 & 59.00 & $39.11 \pm 3.94$ \\
Hemoglobin, g/l & 37.00 & 229.00 & $103.28 \pm 15.06$ \\
Pre-MAP, mm Hg & 47.00 & 151.33 & $97.69 \pm 12.41$ \\
Pre-SBP, mm Hg & 71.00 & 220.00 & $137.25 \pm 20.76$ \\
SBP change, mm Hg & -90.00 & 67.00 & $-9.68 \pm 18.84$ \\
\hline
\end{tabular}

\section{KARGER \\ Fax +41 613061234 \\ E-Mail karger@karger.ch}

www.karger.com

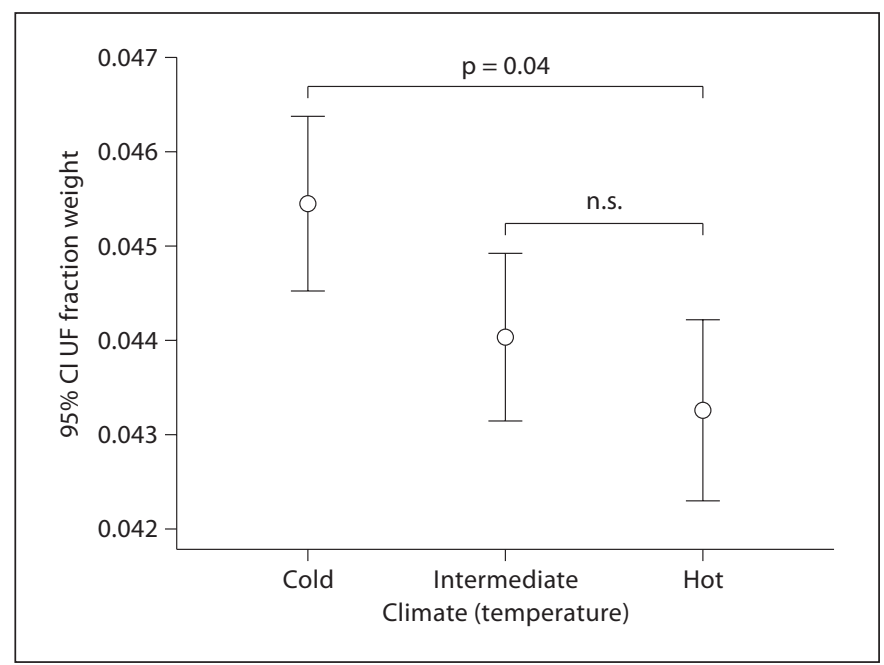

Fig. 1. Mean (95\% CI) for intradialytic fractional weight loss (UF) expressed as fraction of postdialysis weight.

Dr. Hugh Feidhlim Woods

Fresenius Medical Care Asia Pacific

5101 Sun Hun Gai Building

30 Harbour Road, Wanchai, Hong Kong, SAR (China)

Tel. +852 2583 0848, Fax +852 2898 2273, E-Mail feidhlim.woods@fmc-asia.com 

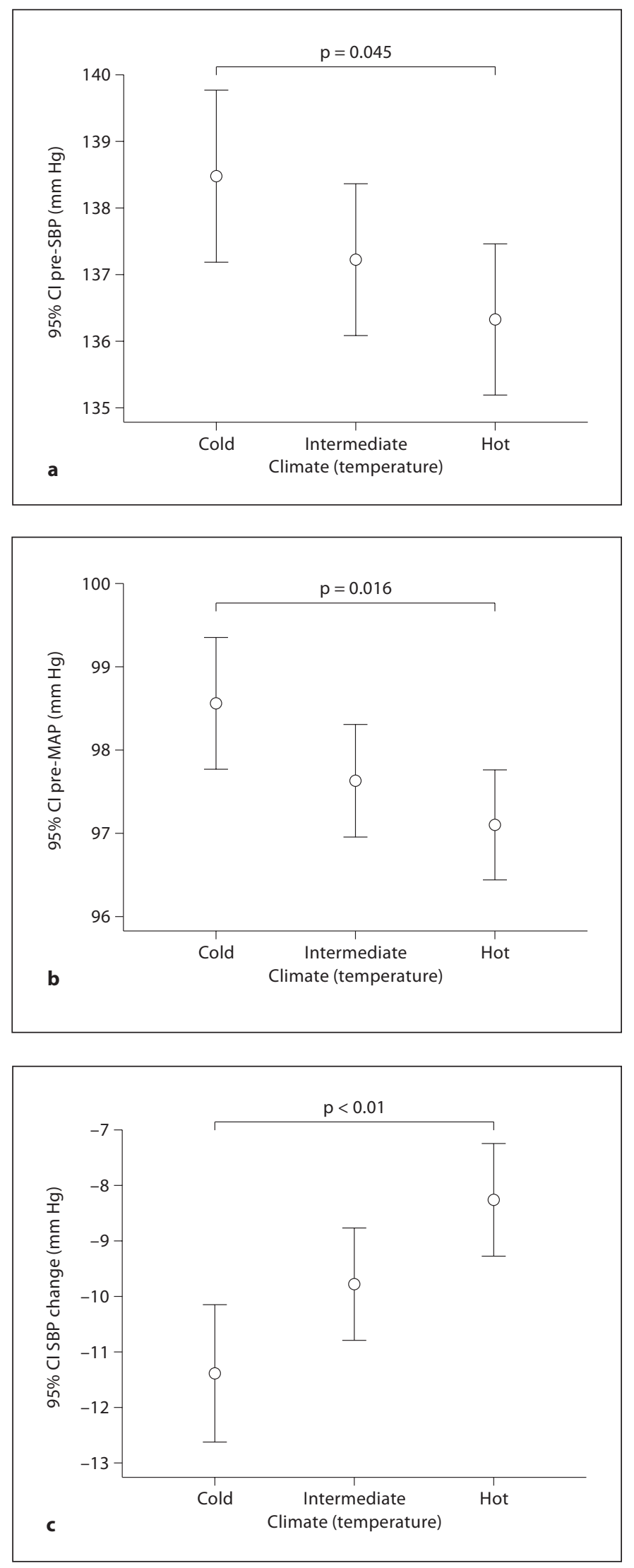

Climate and Volume and Blood Pressure Trends in Taiwanese HD Patients

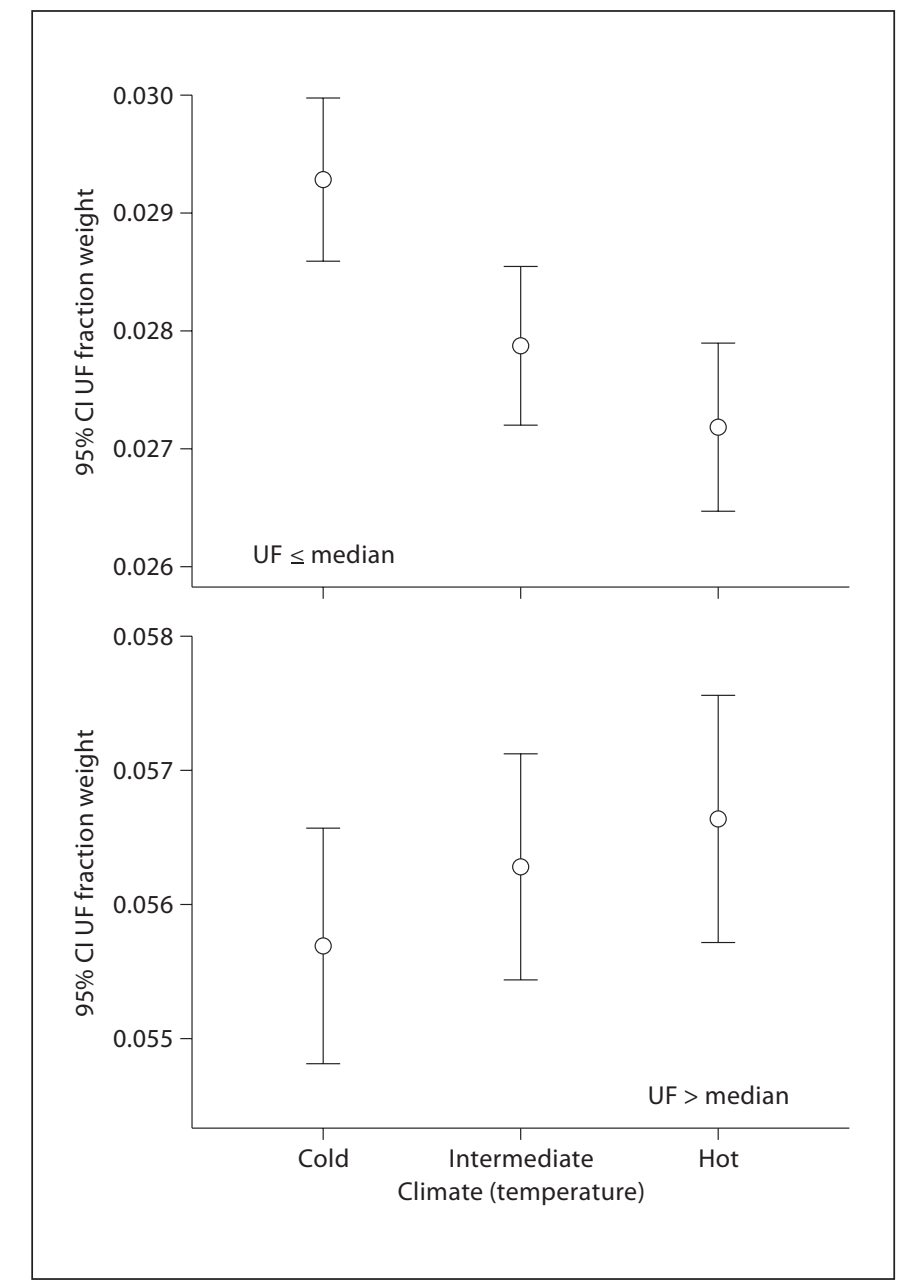

Fig. 3. Mean ( $95 \% \mathrm{CI})$ for fractional weight loss according to whether average UF was $>$ or $\leq$ the median.

Fig. 2. Means $(95 \% \mathrm{CI})$ for predialysis SBP (a), predialysis mean $\mathrm{BP}(\mathbf{b})$ and pre-post change in SBP (c).

Blood Purif 2008;26:404-406 
only evident in those patients with lower ( $\leq$ median) fractional weight loss.

We were not able to identify any difference in these trends comparing diabetic (36\% of the patients) and nondiabetic patients.

We confirm, therefore, seasonal changes in volume status of Taiwanese HD patients and blood pressure correlates with volume status. We would suggest, however, that Hwang fails to consider one important reason for the observed trends. During the cold months in Taiwan, local people consume more soups or broths than during the hot months. For some persons, a broth dish might be consumed at each daily meal thus augmenting salt and fluid intake over that consumed during hotter months. A combination of lower insensible fluid loss and higher intake inadequately compensated for by dialytic fluid removal would explain these findings. Although the differences in fractional weight loss, and by inference, the differences in seasonal fluid gains are small, the trends for the blood pressure (fig. 3) are clinically significant. It would be appropriate to define and moderate seasonal changes in fluid intake through education and counseling.

\section{Reference}

1 Hwang JC, Wang CT, Chien CC: Effect of climatic temperature on fluid gain in hemodialysis patients with difference degrees of over-hydration. Blood Purif 2007;25:473479. 